\title{
Cycle biologique au laboratoire de Plasmodium berghei killicki n. sub.sp.
}

\author{
par I. LANDAU, J.-C. MICHEL et J.-P. ADAM ${ }^{1}$ \\ Laboratoire de Zoologie (Vers) associé au C.N.R.S. ( $\mathrm{P}^{r}$ A.-G. ChaвAud) \\ (Muséum National d'Histoire Naturelle, 57, rue Cuvier, 75 -Paris-5) \\ et Office de la Recherche Scientifique et Technique d'Outre-Mer, Brazzaville (Rép. Congo)

\section{Résumé} \\ Le cycle de Plasmodium berghei killicki n. sub. sp. a été \\ obtenu chez Anopheles stephensi et le Rat blanc. P.b. killicki \\ se distingue essentiellement des deux autres sous-espèces de $P$. \\ berghei par la taille de ses sporozoïtes, le diamètre des oocystes \\ et les modifications du noyau de la cellule hépatique hôte.
}

\section{Summary}

The life cycle of Plasmodium berghei killicki n. sub. sp. was obtained in Anopheles stephensi and the white Rat. P.b. killicki differs from the two other subspecies of $P$. berghei, mainly by the length of the sporozoïtes, the diameter of the oocysts and the alterations in the nucleus of the hepatic host cell.

(1) Travail effectué grâce à une subvention de l'Organisation Mondiale de la Santé. 
Adam, Landau et Chabaud ont découvert en 1966 un foyer de Thamnomys rutilans paludéens dans les environs de Brazzaville.

Comme dans les autres localités connues en Afrique, les Rongeurs sont parasités par deux espèces de Plasmodium. L'une appartient au groupe berghei; elle est rare dans la nature puisque l'examen de nombreux Rongeurs ne nous a permis d'en isoler que deux souches. L'autre beaucoup plus fréquente se rattache au groupe vinckei.

Nos techniques de travail ont été les mêmes que ceiles employées par Landau et Killick-Kendrick (1966) pour l'étude des Plasmodium de Rongeurs de la République Centrafricaine.

Nous avons utilisé :

1) La souche 193 L (1) isolée à partir d'un Thamnomys rutilans capturé en septembre 1966 à N'ganga Lingolo, près de Brazzaville et maintenu en captivité au laboratoire.

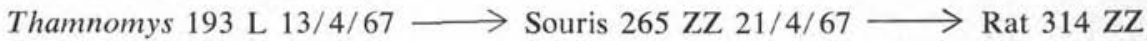

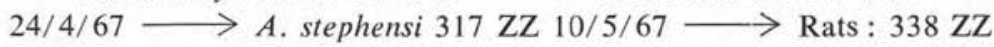

$339 \mathrm{ZZ}$

$340 \mathrm{ZZ}$

$341 \mathrm{ZZ}$

$342 \mathrm{ZZ}$

La souris $265 \mathrm{ZZ}$ inoculée à partir du Thamnomys $193 \mathrm{~L}$ a présenté dans son sang une infection mixte à Plasmodium type berghei et Plasmodium type vinckei. Le passage au Rat $314 \mathrm{ZZ}$ a permis d'isoler le Plasmodium type berghei.

2) Des Anopheles stephensi élevés à la «London School of Hygiene and Tropical Medicine» et au «Muséum National d'Histoire Naturelle». Les moustiques de ces deux é'evages proviennent de la souche d'A. stephensi maintenue par R.P.G. Shute au « Malaria Reference Laboratory » (Epsom).

3) Des rats blancs provenant de la même souche que ceux utilisés pour la description des schizontes pré-érythrocytaires de P.b. yoelii Landau et Killick-Kendrick 1966.

\section{Sporogonie.}

Le moment le plus favorable pour gorger les Anophèles sur des Rats et des Souris porteurs de gamétocytes, se situe entre le troisième et le sixième jour après l'inoculation de sang infectant à ces Rongeurs. Dans ces conditions, le taux d'infection des Moustiques est en général supérieur à $70 \%$.

Les Anophèles de la cage $317 \mathrm{ZZ}$ ont été gorgés sur le Rat $314 \mathrm{ZZ}$, le troisième jour après son inoculation par du sang; on observait alors de nombreux gamétocytes dans les frottis. La cage a été placée ensuite dans un insectarium maintenu entre $22^{\circ}$

(1) Nous ne précisons que la généalogie de la souche qui nous a permis de décrire la schizogonie pré-érythrocytaire. Cette souche a aussi été utilisée après plusieurs passages sur Anopheles stephensi et sur Rongeurs, en particulier pour l'étude de la sporogonie. Les résultats furent comparables dans toutes les expériences. 
et $24^{\circ}$. Dans cette expérience, le nombre d'oocystes a varié de 50 à environ 300 . Comme chez les autres Plasmodium du groupe berghei, on trouve dans un même estomac des oocystes à des stades de développement très différents.

Le pigment dans les oocystes jeunes est identique à celui de P.b. yoelii. Les premiers oocystes contenant des sporozoïtes sont observés le huitième jour et la taille des oocystes mûrs atteint $60 \mu$. Les sporozoïtes font leur première apparition dans les glandes salivaires les dixième et onzième jours. La longueur, mesurée sur 20 sporozoïtes dans des préparations séchées à l'air et fixées à l'alcool méthylique, est de $18 \mu(15,5 \mu$ $20,15 \mu)$.

\section{Schizogonie pré-érythrocytaire.}

Des jeunes Rats de un mois ont été inoculés chacun avec 32 à 40 glandes salivaires d'A. stephensi $317 \mathrm{ZZ}$, puis sacrifiés à $30,36,45$ et 50 heures. Leur foie renfermait un schizonte pré-érythrocytaire toutes les cina à quinze coupes. La taille moyenne des deux schizontes pré-érythrocytaires à 30 heures est de $13 \mu$; de cinq schizontes à 36 heures : $18 \mu$; de onze schizontes à 45 heures : $32 \mu$, et de treize schizontes à 50 heures : $34,5 \mu$. Le sang est devenu positif pour la première fois à la $46^{\circ}$ heure. Le premier schizonte mûr contenant des mérozoites a été trouvé à 50 heures.

Dans l'ensemble, les schizontes pré-érythrocytaires présentent peu de différences morphologiques avec ceux de P.b. yoelii.

Les formes jeunes mesurent $14,8 \times 11,2 \mu(15,5-14 \times 11-12,4 \mu)$ à 30 heures, et $19,8 \times 16,2 \mu(21,7-18,6 \mu \times 18,5-15,5 \mu)$ à 36 heures, et comme chez P.b.yoelii elles ont souvent des noyaux de grande taille, irréguliers avec une chromatine disposée en couronne.

A 45 heures, leur aspect morphologique est plus varié. Leur taille est de $36,1 \times$ $28,4 \mu(29,5-46,5 \mu \times 23,2-31 \mu)$. La forme la plus fréquente est celle que l'on rencontre à la même heure chez P.b. yoelii : arrondie, ou ovalaire, à noyaux petits et uniformément répartis dans un cytoplasme bleu foncé contenant des aggrégats très basophiles. Certains schizontes, cependant, renferment des noyaux plus grands, disposés en palissade à la périphérie et contiennent des pseudo-cytomères; comme chez P.b. yoelii, ils sont souvent envahis par des macrophages. D'auties formes plus particulières n'ont jamais été observées chez P.b. yoelii ; leur cytoplasme est peu dense, très vacuolé et leurs noyaux sont grands avec une disposition en couronne, de la chromatine analogue à celle que l'on trouve à 30 et 36 heures. Par leur petite taille, leur cytoplasme peu abondant et leurs noyaux en couronne, ils rappellent les schizontes hépatiques secondaires trouvés chez des Thamnomys rutilans paludéens capturés en République Centrafricaine (Landau, Chabaud, Adam, Michel et Boulard 1968). Nous n'avons cependant noté ni capsule, ni nucléole. La plupart des noyaux de la cellule hépatique hôte sont très augmentés de taille, leur chromatine est rassemblée à la périphérie, laissant au centre un espace clair plus ou moins étendu.

A 50 heures, les schizontes pré-érythrocytaires mesurent $38,3 \times 30,7 \mu(31-48 \mu$ 


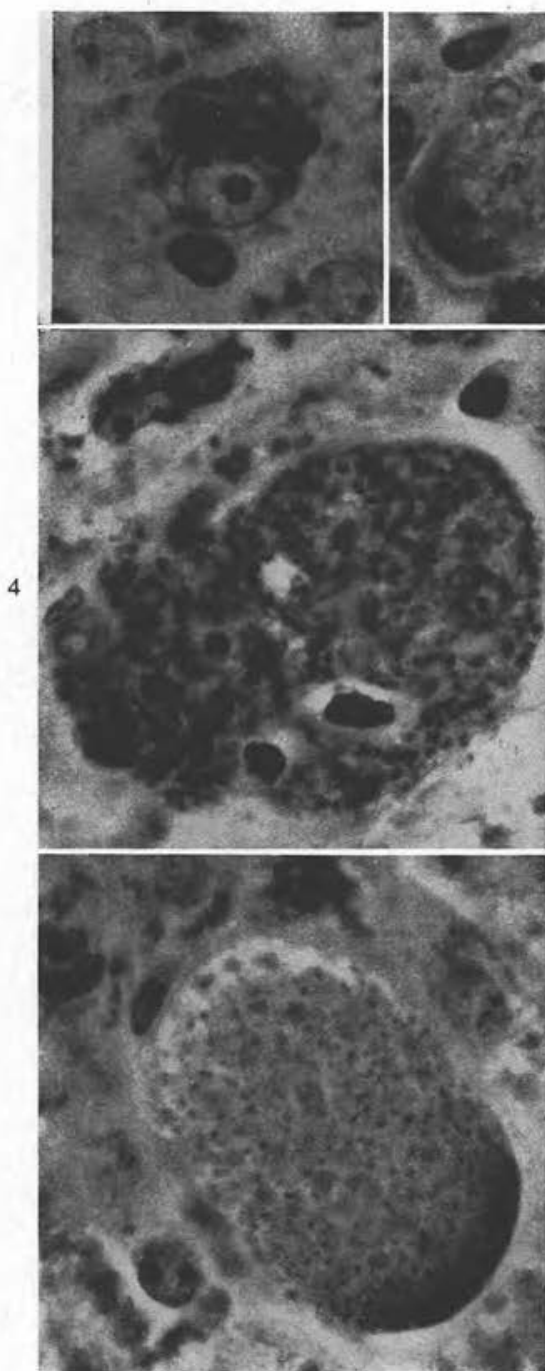

6
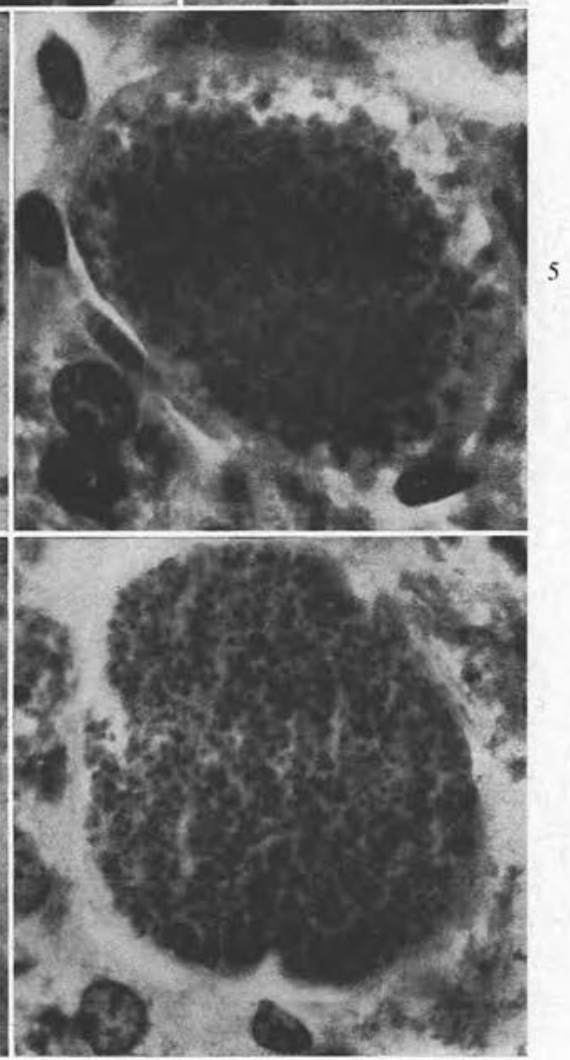

1. - Schizonte âgé de 30 heures

2. - Schizonte âgé de 45 heures

présentant une disposition en couronne de la chromatine nucléaire 3. - Même type de schizonte que le précédent après hydrolyse 4. - Schizonte âgé de 45 heures avec pseudo-cytomères 5 et 6 . - Schizonte âgé de 50 heures 7. - Schizonte mûr âgé de 50 heures 
$\times 23-42 \mu$ ). La plupart sont en tous points comparables à ceux de P.b. yoelii. On retrouve quelques-unes des formes particulières observées à 45 heures. Sur 50 schizontes examinés, nous n'avons vu qu'une seule forme mûre. Le noyau de la cellule hépatique présente les mêmes modifications qu'à 45 heures, souvent plus accentuées. Le nombre approximatif de noyaux d'un schizonte mûr à 50 heures est de 8.000 .

\section{Discussion.}

Les caractéristiques du cycle des autres sous-espèces du groupe berghei ont été précisées par Yoeli (1965), Landau et Killick-Kendrick (1966) et Wery (1967).

L'étude du cycle biologique de la souche de Brazzaville révèle deux caractères importants et quelques caractères mineurs qui permettent de la différencier de P.b. berghei et de $P . b$. yoelii. Nous estimons qu'il s'agit d'une sous-espèce de $P$. berghei distincte des deux sous-espèces existantes et nous la nommons Plasmodium berghei killicki $\mathrm{n}$. sub. sp.

P.b. killicki se distingue des autres sous-espèces de $P$. berghei par les caractères suivants :

1) la taille des sporozoïtes qui mesurent $18 \mu$ de longueur en moyenne, alors que ceux de P.b. berghei ont $12 \mu$ et ceux de P.b. yoelii, $14 \mu$;

2) le diamètre des oocystes mûrs qui est de $60 \mu$ en moyenne, alors que chez P.b. berghei, il est de $40 \mu$ et chez P.b. yoelii de $80 \mu$;

3) les modifications du noyau de la cellule hépatique, jamais observées chez P.b. berghei, rares et peu marquées chez P.b. yoelii.

En outre, P.b. killicki se distingue de P.b. yoelii par la durée de son cycle préérythrocytaire supérieure à 43 heures. De P.b. berghei, il diffère par la plus grande taille des schizontes pré-érythrocytaires à tous les stades et la température plus élevée (22 à $\left.24^{\circ}\right)$ à laquelle s'effectue sa sporogonie dans les conditions expérimentales.

Nous remercions vivement le $\mathrm{P}^{r}$ P. C. C. Garnham qui nous a permis de réaliser dans son laboratoire de la «London School of Hygiene and Tropical Medicine » quelques-unes des infections expérimentales mentionnées dans ce travail et qui nous apporte de façon continue l'aide la plus efficace et les conseils les plus précieux.

\section{Bibliographie}

Adam (J.-P.), Landau (I.). et Chabaud (A.-G.), 1966. - Découverte dans la tégion de Brazzaville de Rongeurs infectés par des Plasmodium C. R. Acad. Sci. Paris, 263, p. 140.

Landau (I.), Chabaud (A.-G.), Adam (J.-P.), Michel (J.-C.) et Boulard (Y.), 1968. - Morphologie et évolution des schizontes hépatiques secondaires dans le Paludisme spontané des Rongeurs de La Maboké. Cahiers de La Maboké, 6, p. 63.

Landau (I.) et Killick-Kendrick (R)., 1966. - Rodent Plasmodium of the Republique Centrafricaine. The sporogony and the tissue stages of Plasmodium chabaudi and P. berghei yoelii, Trans. R. Soc. Trop. Med. Hyg., 60, p. 633.

WERY (M.), 1967. - Studies on the sporogony of Rodent Malaria. Ph. D. Thesis, University of London.

Yoeli (M.), 1965. - Studies on Plasmodium berghei in nature and under experimental conditions. Trans. R. Soc. Trop. Med. Hyg., 59, p. 255. 